\title{
On the Rate-dependent Plasticity Modelling of Unidirectional Fibre-reinforced Polymeric Matrix Composites
}

\author{
Borja Erice ${ }^{*}$, Daniel Thomson, Sathiskumar A. Ponnusami, Mehtab V. Pathan, and Nik Petrinic \\ Department of Engineering Science, University of Oxford, Parks Road, Oxford, OX1 3PJ, United Kingdom Department
}

\begin{abstract}
Three different approaches to plasticity are investigated to model the experimentally-observed non-linear behaviour of unidirectional fibre-reinforced polymeric matrix materials. The first and simplest approach consists on assuming independent one-dimensional rate-dependent plasticity on in-plane (12) and through-thickness longitudinal (13) shear components of the Cauchy stress tensor. The second, employs a 3D extension of the plane stress Hill' 48 anisotropic plastic surface. The third and the last is formulated as a quadratic yield function inspired by Puck's fracture initiation criterion. It searches for a plastic localisation plane in which a certain combination of normal and shear stresses is maximum. Numerical simulations are conducted to analyse the off-axis compression behaviour of carbon fibre reinforced epoxy composite under varying loading rates. The afore-mentioned three different approaches are explored with an aim to predict the experimentally-observed non-linear response of such composites. The model parameters are determined using a deterministic inverse modelling strategy employing an iterative domain reduction optimisation technique. As far as the experiments are concerned, the quasi-static and medium rate tests were carried out in universal testing machines, while the experiments at high rate were conducted in a Split Hopkinson Pressure Bar system. The effectiveness in terms of accuracy and robustness of the three approaches are discussed.
\end{abstract}

\section{Introduction}

The experimentally observed non-linearity on the stressstrain behaviour of Unidirectional (UD) Fibre Reinforced Polymers (FRP) is a topic were many have tried to tackle. However, due to the heterogeneous nature of the material and differences in which physically-based continuum theory to choose, it has not arisen any agreement on how to treat such non-linearity. Issues like this have been settled long time ago in other types of materials such as metals, where the plasticity theories are well established. Furthermore, models that deal with other physical aspects of the material such as strain dependency are most of the times not taken into account. Several models have been proposed during the last years to address the facts exposed in the previous paragraph. Some as Goldberg et al. in [1-2] have developed a 1D shear plasticity models that allow for the input of piecewise stress-strain curves. Such model is already available in LS-DYNA non-linear finite element code as MAT_213. Other as Vyas et al. [3] have tried more convincing 3D approaches that include the Raghava pressure-dependent plasticity function combined with a null flow rule component in the fibre direction. Such approach was previously proposed by Xie and Adams [4]. A more practical approach has been proposed by Puck in [5], where he basically scales the non-linear shear stress-strain curves according to his own physically-based two-dimensional inter fibre fracture criterion.

Based on the research performed until now and some new approaches we will compare the predictive capabilities of three different models to simulate the nonlinear behaviour of UD FRPs. The following models will be compared against some off-axis compression stressstrain data found in the literature:

- 1D plasticity in 12 and 13 shear components,

- Three-dimensional extension of the Hill'48 plane stress anisotropic plasticity,

- Localisation plane-based plasticity as described in [6].

All three will be combined with Voce-type isotropic strain hardening and strain rate hardening.

First we will describe the numerical models used. The next section will focus on the numerical simulations performed in order to compare them against experimental tests. After that we will discuss the results and make some concluding remarks.

\section{Elasticity}

The elastic orthotropic law relates these Cauchy stress $\sigma$ and strain $\varepsilon$ vectors as follows: 


$$
\boldsymbol{\sigma}=\mathbf{C} \boldsymbol{\varepsilon}^{e}=\mathbf{C}\left(\boldsymbol{\varepsilon}-\boldsymbol{\varepsilon}^{p}\right)
$$

where $\boldsymbol{\varepsilon}^{e}$ and $\boldsymbol{\varepsilon}^{p}$ are the elastic and the plastic parts of the strain vector and $\mathbf{C}$ is a $6 \times 6$ symmetric matrix that contains the orthotropic elastic constants:

$$
\begin{array}{cc}
C_{11}=E_{11}\left(1-v_{23} v_{32}\right) C & C_{12}=E_{11}\left(v_{21}+v_{31} v_{23}\right) C \\
C_{22}=E_{22}\left(1-v_{13} v_{31}\right) C & C_{13}=E_{11}\left(v_{31}+v_{21} v_{32}\right) C \\
C_{33}=E_{33}\left(1-v_{12} v_{21}\right) C & C_{23}=E_{22}\left(v_{32}+v_{12} v_{31}\right) C \\
C_{44}=G_{12}, C_{55}=G_{23}, C_{66}=G_{13}
\end{array}
$$

being,

$$
C=\frac{1}{1-v_{12} v_{21}-v_{23} v_{32}-v_{13} v_{31}-2 v_{21} v_{32} v_{13}}
$$

The matrix symmetry imposes additional constraints that reduces the number of independent elastic constants from nine to five,

$$
\frac{v_{i j}}{E_{i i}}=\frac{v_{j i}}{E_{j j}} \text { for } i=\{1,2,3\}
$$

Please note that the fibre orientation is assumed to coincide with the $\mathbf{e}_{1}$ direction of the local material coordinate system $\left\{\mathbf{e}_{1}, \mathbf{e}_{2}, \mathbf{e}_{3}\right\}$ (see Fig. 1).

The rate-dependent stiffness is modelled using a phenomenological law based on results found in the literature [7]. The fibre direction is assumed to be rateindependent, while the rest of the elastic moduli are given by:

$$
\begin{array}{ll}
E_{22}=E_{22}^{0}\left(1+\sqrt{r_{\mathrm{c}} \dot{\varepsilon}_{22}}\right) & G_{12}=G_{12}^{0}\left(1+\sqrt{r_{\mathrm{c}} \dot{\gamma}_{12}}\right) \\
E_{33}=E_{33}^{0}\left(1+\sqrt{r_{\mathrm{c}} \dot{\varepsilon}_{33}}\right) & G_{23}=G_{23}^{0}\left(1+\sqrt{r_{\mathrm{c}} \dot{\gamma}_{23}}\right) \\
G_{13}=G_{13}^{0}\left(1+\sqrt{r_{\mathrm{c}} \dot{\gamma}_{13}}\right)
\end{array}
$$

being the rate-dependent coefficient for the stiffness matrix.

\section{Modelling of the non-linear behaviour}

\subsection{D plasticity}

In this case, a simple one-dimensional rate-dependent plasticity is adopted to model the non-linear behaviour of the in-plane and transverse shear $(i=\{12,13\})$. The ratedependent yield function is defined as,

$$
f\left[\tau_{(i)}, \bar{\gamma}_{(i)}^{p}, \dot{\bar{\gamma}}_{(i)}^{p}\right]=\left|\tau_{(i)}\right|-k\left[\bar{\gamma}_{(i)}^{p}, \dot{\bar{\gamma}}_{(i)}^{p}\right]=0
$$

being $k$ the strain hardening defined as a function of the equivalent plastic shear strain $\bar{\gamma}_{(i)}^{p}$ and equivalent shear strain rate $\dot{\bar{\gamma}}_{(i)}^{p}$. The flow rule is given by,

$$
\dot{\gamma}_{(i)}^{p}=\dot{\lambda} \frac{\partial f}{\partial \tau_{(i)}}=\dot{\lambda} \operatorname{sign}\left[\tau_{(i)}\right]
$$

$$
\dot{\bar{\gamma}}_{(i)}^{p}=\left|\dot{\gamma}_{(i)}^{p}\right|=\dot{\lambda} \text { being } \bar{\gamma}_{(i)}^{p}=\int \dot{\bar{\gamma}}_{(i)}^{p} d t=\lambda
$$

The Wang or consistency model [8] is used here to

$$
\begin{aligned}
\dot{f} & =\frac{\partial f}{\partial \tau_{(i)}} \dot{\tau}_{(i)}-\frac{\partial k}{\partial \bar{\gamma}_{(i)}^{p}} \dot{\bar{\gamma}}_{(i)}^{p}-\frac{\partial k}{\partial \dot{\bar{\gamma}}_{(i)}^{p}} \ddot{\bar{\gamma}}_{(i)}^{p} \\
& =\operatorname{sign}\left[\tau_{(i)}\right] \dot{\tau}_{(i)} \dot{\lambda}-H_{s(i)} \dot{\lambda}-H_{d(i)} \ddot{\lambda}=0
\end{aligned}
$$

where $H_{s(i)}=\partial k / \partial \bar{\gamma}_{(i)}^{p}$ and $H_{d(i)}=\partial k / \partial \dot{\bar{\gamma}}_{(i)}^{p}$.

For the sake of completeness the loading/unloading conditions are also given:

$$
f \leq 0, \quad \dot{\lambda} \geq 0, \quad \dot{\lambda} f=0
$$

Please note that such conditions are applicable to the rest of the plasticity models. The yield function expressed as a function of the stress vector $\boldsymbol{\sigma}$, the equivalent plastic strain $\bar{\varepsilon}_{p}$ and equivalent plastic strain rate $\dot{\bar{\varepsilon}}_{p}$ reads:

$$
f\left[\boldsymbol{\sigma}, \bar{\varepsilon}_{p}, \dot{\bar{\varepsilon}}_{p}\right]=\bar{\sigma}_{h}[\boldsymbol{\sigma}]-k\left[\bar{\varepsilon}_{p}, \dot{\bar{\varepsilon}}_{p}\right]=0
$$

\subsection{Hill'48 anisotropic plasticity}

A modified 3D extension of the rate-dependent Hill'48 plane stress yield function is proposed this time to model the plastic response of the UD-reinforced fibrereinforced where $\bar{\sigma}_{h}=\sqrt{\boldsymbol{\sigma}^{T} \mathbf{P} \boldsymbol{\sigma}}$ is equivalent Hill stress, $k$ is the isotropic hardening and $\mathbf{P}$ is a symmetric matrix that can be expressed as,

$$
\mathbf{P}[\theta]=\left[\begin{array}{cccccc}
P_{11}[\theta] & P_{12} & -\left(1+P_{12}\right) & 0 & 0 & 0 \\
P_{12} & P_{22} & -\left(P_{12}+P_{22}\right) & 0 & 0 & 0 \\
-\left(1+P_{12}\right) & -\left(P_{12}+P_{22}\right) & 1+2 P_{12}+P_{22} & 0 & 0 & 0 \\
0 & 0 & 0 & P_{33} & 0 & 0 \\
0 & 0 & 0 & 0 & 3 & 0 \\
0 & 0 & 0 & 0 & 0 & 3
\end{array}\right]
$$

It contains Hill's planar anisotropic weighting constants $P_{11}[\theta], P_{22}, P_{12}$ and $P_{33}$. Please note that the yield function will collapse into a von Mises material if $P_{11}=P_{22}=1, P_{12}=-0.5$ and $P_{33}=3$ being $\mathbf{P}=\mathbf{P}_{\mathrm{VM}} \cdot \theta$ is the angle between the fibre orientation and the load carrying axis. Mathematically speaking, it is defined as $\theta=\cos ^{-1}\left[\mathbf{e}_{1} \cdot \mathbf{i}\right]$, being $\mathbf{i}$ the global $\mathrm{x}$ axis unit vector as shown in Fig. 1. Such angle is extracted from the fibre rotation matrix $\mathbf{R}_{f}=\mathbf{R}_{t} \mathbf{R}_{r}^{T}$ as in [9]. The total rotation is obtained from the polar decomposition of the deformation gradient expressed in global coordinates as:

$$
\mathbf{R}_{t}=\mathbf{F U}^{-1}
$$

and the rigid body rotation is calculated from the spin tensor $\mathbf{W}=\operatorname{skew}[\mathbf{L}]$ as:

$$
\dot{\mathbf{R}}_{r}=\mathbf{W R}_{r}
$$

being the $\mathbf{L}$ velocity gradient. For more details on the fibre rotation approach and how it affects to the strain and stress tensors the reader is referred to [9].

where $\dot{\lambda}$ is the plastic multiplier defined as follows, 


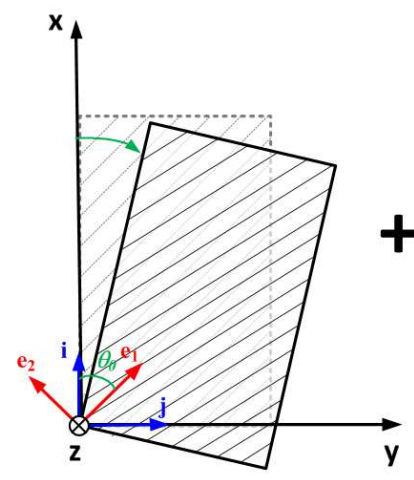

Rigid body rotation $R_{r}$

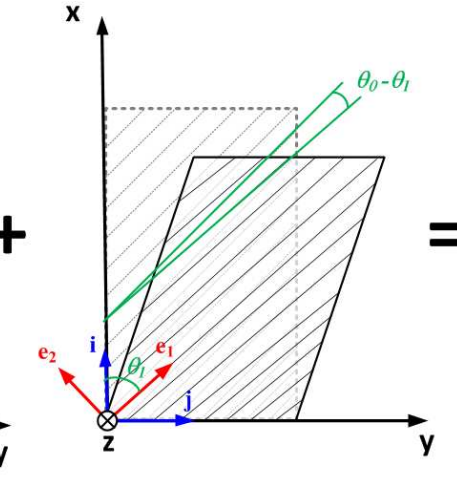

Fibre rotation $\mathbf{R}_{\mathbf{f}}$

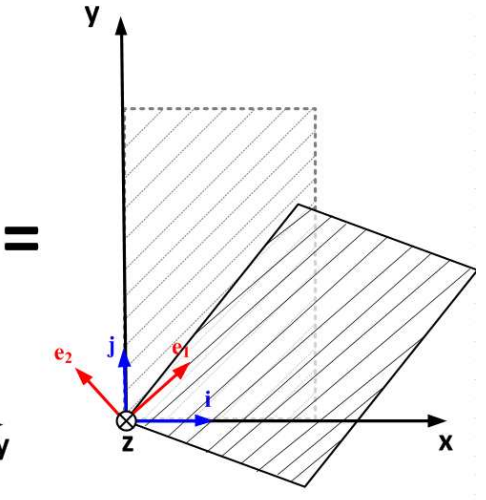

Total rotation $R_{\mathrm{t}}$

Fig. 1. Decomposition of the total rotation in the rigid body rotation and the fibre rotation.

Since the 0 degree orientation is assumed to the purely elastic, if $P_{11}=p_{1} \sin ^{p_{2}}[\theta], P_{11}$ is zero when $\theta=0^{\circ}$ and $p_{1}$ when $\theta=0^{\circ} . p_{2}$ allows for finer tuning of the intermediate stages between both angles.

The flow rule is chosen to be associative and is given by,

$$
\dot{\boldsymbol{\varepsilon}}^{p}=\dot{\lambda} \frac{\partial f}{\partial \boldsymbol{\sigma}}=\dot{\lambda} \frac{\mathbf{P} \boldsymbol{\sigma}}{\bar{\sigma}_{h}}
$$

From the work conjugacy, $\boldsymbol{\sigma}^{T} \dot{\boldsymbol{\varepsilon}}^{p}=\bar{\sigma}_{h} \dot{\bar{\varepsilon}}_{p}$, one gets the evolution equation of the equivalent plastic strain that relating it with the plastic multiplier $\dot{\lambda}$ as,

$$
\dot{\bar{\varepsilon}}_{p}=\dot{\lambda} \text { being } \bar{\varepsilon}_{p}=\int \dot{\bar{\varepsilon}}_{p} d t
$$

Following the Wang or consistency model [8] the yield function is differentiated to get the consistency condition ( $\dot{f}=0$ ) in terms of the internal hardening variables $\bar{\varepsilon}_{p}$ and $\dot{\bar{\varepsilon}}_{p}$. This gives the following second order differential equation on $\lambda$,

$$
\begin{aligned}
\dot{f} & =\frac{\partial f}{\partial \boldsymbol{\sigma}} \dot{\boldsymbol{\sigma}}-\frac{\partial k}{\partial \bar{\varepsilon}_{p}} \dot{\bar{\varepsilon}}_{p}-\frac{\partial k}{\partial \dot{\bar{\varepsilon}}_{p}} \ddot{\bar{\varepsilon}}_{p} \\
& =\dot{\boldsymbol{\sigma}}^{T} \mathbf{m} \dot{\lambda}-H_{s} \dot{\lambda}-H_{d} \ddot{\lambda}=0
\end{aligned}
$$

where $H_{s}=\partial k / \partial \bar{\varepsilon}_{p}$ and $H_{d}=\partial k / \partial \dot{\bar{\varepsilon}}_{p}$.

\subsection{Localisation plane-based plasticity}

The yield function, originally reported in [6], that is proposed for the localisation plane (LP) plasticity model is expressed as:

$$
f\left[\mathbf{r}, \bar{\varepsilon}_{p}, \dot{\bar{\varepsilon}}_{p}\right]=\bar{\sigma}_{\mathrm{lp}}[\mathbf{r}]-k\left[\bar{\varepsilon}_{p}, \dot{\bar{\varepsilon}}_{p}\right]=0
$$

where $\bar{\sigma}_{\mathrm{lp}}=\alpha_{f} \sigma_{n}+\sqrt{\tau_{n 1}^{2}+\beta \tau_{n t}^{2}}$ is the localisation plane equivalent stress, $\alpha_{f}$ is the friction coefficient and $\beta$ is the elliptical shear interaction parameter. Instead of using the Cauchy stress vector, we employ the localisation plane reduced stress vector $\mathbf{r}$ that is comprised only by the stress components that affect to such plane: the normal stress $\sigma_{n}$ and the two components of the shear stress; the longitudinal $\tau_{n 1}$ and transversal $\tau_{n t}$. The reduced stress vector

$$
\mathbf{r}=\left\{\begin{array}{lll}
\sigma_{n} & \tau_{n 1} & \tau_{n t}
\end{array}\right\}^{T}=\left\{\begin{array}{lll}
\hat{\sigma}_{2} & \hat{\sigma}_{4} & \hat{\sigma}_{5}
\end{array}\right\}^{T}
$$

is obtained by rotating the Cauchy stress vector into the localisation plane (see Fig. 2) as $\hat{\boldsymbol{\sigma}}=\mathbf{R}_{1} \boldsymbol{\sigma}$ where

$$
\begin{aligned}
\mathbf{R}_{1} & =\left[\begin{array}{cccccc}
1 & 0 & 0 & 0 & 0 & 0 \\
0 & \mathrm{c}^{2} & \mathrm{~s}^{2} & 0 & 2 \mathrm{cs} & 0 \\
0 & \mathrm{~s}^{2} & \mathrm{c}^{2} & 0 & -2 \mathrm{cs} & 0 \\
0 & 0 & 0 & \mathrm{c} & 0 & \mathrm{~s} \\
0 & -\mathrm{cs} & \mathrm{cs} & 0 & \mathrm{c}^{2}-\mathrm{s}^{2} & 0 \\
0 & 0 & 0 & -\mathrm{s} & 0 & \mathrm{c}
\end{array}\right] \\
\mathrm{s}^{n} & =\sin ^{n}\left[\theta_{f r}\right] \quad \mathrm{c}^{n}=\cos ^{n}\left[\theta_{f r}\right]
\end{aligned}
$$

In the same fashion, the rotated strain rate vector is:

$$
\dot{\hat{\boldsymbol{\varepsilon}}}=\mathbf{R}_{1} \dot{\boldsymbol{\varepsilon}}
$$

which leads to a reduced strain rate vector $\dot{\mathbf{e}}$ that has the following components:

$$
\dot{\mathbf{e}}=\left\{\begin{array}{lll}
\dot{\hat{\varepsilon}}_{2} & \dot{\hat{\varepsilon}}_{4} & \dot{\hat{\varepsilon}}_{5}
\end{array}\right\}^{T}=\left\{\begin{array}{lll}
\dot{\varepsilon}_{n} & \dot{\gamma}_{n 1} & \dot{\gamma}_{n t}
\end{array}\right\}^{T}
$$

The flow rule is chosen to be non-associative as generally is in most of the pressure-dependent plasticity models. The plastic potential is:

$$
g[\mathbf{r}]=\alpha_{g} \sigma_{n}+\sqrt{\tau_{n 1}^{2}+e \tau_{n t}^{2}}
$$

The directions of the reduced plastic strains are given by:

$$
\dot{\mathbf{e}}^{p}=\dot{\lambda} \frac{\partial g}{\partial \boldsymbol{\sigma}}=\dot{\lambda} \mathbf{m}
$$

where the flow vector $\mathbf{m}$ is:

$$
\mathbf{m}=\left\{\alpha_{g} \frac{\tau_{n 1}}{\sqrt{\tau_{n t}^{2}+e \tau_{n t}^{2}}} \frac{e \tau_{n t}}{\sqrt{\tau_{n t}^{2}+e \tau_{n t}^{2}}}\right\}^{T}
$$

As in the previous section the relationship between the plastic multiplier and the equivalent plastic strain is obtained from the work conjugacy, in this case, $\mathbf{r}^{T} \dot{\mathbf{e}}^{p}=\bar{\sigma}_{\mathrm{lp}} \dot{\bar{\varepsilon}}_{p}$ that yields to the following relationship,

$$
\dot{\bar{\varepsilon}}_{p}=\dot{\lambda} \frac{\mathbf{r}: \mathbf{m}}{\bar{\sigma}_{\mathrm{lp}}} \text { being } \bar{\varepsilon}_{p}=\int \dot{\bar{\varepsilon}}_{p} d t
$$

The consistency condition $(\dot{f}=0)$ in terms of the internal variables gives: 


$$
\begin{aligned}
\dot{f} & =\frac{\partial f}{\partial \mathbf{r}} \dot{\mathbf{r}}-\frac{\partial k}{\partial \bar{\varepsilon}_{p}} \dot{\bar{\varepsilon}}_{p}-\frac{\partial k}{\partial \dot{\bar{\varepsilon}}_{p}} \ddot{\bar{\varepsilon}}_{p} \\
& =\dot{\mathbf{r}}^{T} \mathbf{m} \dot{\lambda}-H_{s} \dot{\lambda}-H_{d} \ddot{\lambda}=0
\end{aligned}
$$

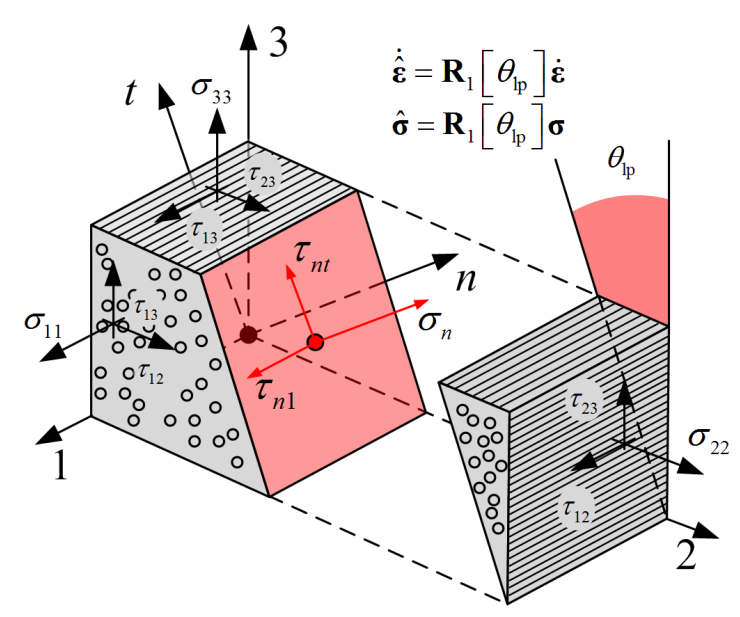

Fig. 2. Schematic view of the rotation performed to transform the stresses into the localisation plane.

\section{Isotropic hardening}

The hardening of the material is modelled as purely isotropic. For the sake of simplicity, no kinematic or distortional hardening is considered. In order to do so, a combined Voce strain hardening and strain rate hardening is defined as a function of the equivalent plastic strain and equivalent plastic strain rate as;

$$
\begin{aligned}
& k\left[\bar{\varepsilon}_{p}, \dot{\bar{\varepsilon}}_{p}\right]=k_{s}\left[\bar{\varepsilon}_{p}\right] k_{d}\left[\dot{\bar{\varepsilon}}_{p}\right] \\
& =\left\{\sigma_{0}+\sum_{i}^{2} Q_{i}\left(1-\exp \left[-C_{i} \bar{\varepsilon}_{p}\right]\right)\right\}\left\{1+\sqrt{k_{p} \dot{\bar{\varepsilon}}_{p}}\right\}
\end{aligned}
$$

where $k_{p}$ is the strain rate coefficient, $\sigma_{0}$ is the yield stress and $\left\{Q_{i}, C_{i}\right\}$ are the strain hardening material constants. The strain hardening and the strain rate hardening effects have been considered independent for the sake of simplicity and ease of calibration. This approach has been widely used in rate-dependent metal plasticity [10-12].

\section{Numerical simulations}

The objective of this investigation was to compare the three different approaches presented so far to model the non-linearity observed in the UD FRPs. In order to do so, the stress-strain curves of off-axis compression tests performed under quasi-static and dynamic loading conditions were compared with numerical simulations carried out with finite element analysis. The tests, reported in [13] were conducted obtaining off-axis prismatic specimens from UD IM7/8552 material system laminates.

The three approaches described in the previous sections were implemented in the non-linear finite element code LS-DYNA. As an initial step towards a fully developed comparison, the simplest of the simulations was conceived: a single element simulation. In general, the level of strain field uniformity that the specimens obtained from UD laminates present under uniaxial loading conditions makes this type of approach more forgiving than in other types of materials such as ductile metals or polymers. The single element was loaded by the prescribed movement of frictionless rigid walls with particular displacement $(u[t])$ boundary conditions that could be obtained from the detailed information in form of axial strain-time plots in [13] as $u[t]=L_{0} \varepsilon_{\text {ax }}[t], L_{0}$ where is the length of the element. The elastic constants were obtained from one of the widely available literature on the studied material system [14]. The yield function constants as well as the hardening parameters were calculated through an inverse modelling strategy employing an iterative domain reduction optimisation available in the software package LS-OPT. The material constants used to perform the numerical simulations at quasi-static and dynamic regimes from which stress-strain curves shown in Fig.3 and Fig. 4 respectively are reported in Tables 1 to 4 .

\section{Discussion}

From Fig.3 (a) we can obviously see that the transverse compression behaviour could not be matched by the 1D plasticity model since the model assumed that it was fully elastic. The quasi-static response of the specimens with fibre orientations of $45^{\circ}$ and $60^{\circ}$ was reasonably captured given the simplicity of the model. However, the compressive behaviour of the $30^{\circ}$ specimen was underpredicted. The other two approaches used to model the non-linearity of the material, i.e. Hill' 48 and LP plasticity, seemed to capture with excellent accuracy the off-axis compressive response of the UD IM7/8552 material system as can be seen in Fig.3 (b) and (c).

The comparisons between the experiments conducted at high strain rate with the numerical simulations carried out with the three different approaches are gathered in Fig.4. Despite the lack of accuracy of the 1D plasticity model in predicting the quasi-static response of the material, it seemed to be able to reasonably predict the stress-strain curves of it under dynamic loading conditions. The Hill' 48 and LP plasticity were able to accurately model the stress-strain response for the specimens tested at $60^{\circ}$ and $90^{\circ}$ orientations. Even though, the large majority of the stress-strain curve of the $30^{\circ}$ and $45^{\circ}$ specimens was very well matched the last part of it was underpredicted with both plasticity approaches. Since in this type of materials the thermal softening effects are almost negligible, makes difficult to justify the sudden drop observed experimentally on the hardening of the material. In order to double check such a behaviour, some preliminary in-house tests on prismatic specimens with the same orientations and material were performed under similar loading conditions (see [15] for some more details). The stressstrain curves obtained from such tests are depicted in Fig.5 with solid markers. These, do not present the previously mentioned drop in the stress-strain curves. 
It should be noted that for the sake of consistency the material constants were fitted with the data obtained from the literature. However, the newly performed tests are in better agreement with the models than the ones reported in [13]. Coupon level calibration may shed some light regarding the difference on the hardening present on specimens at $30^{\circ}$ and $45^{\circ}$. The single element calculations are way too simplistic to be able to extract definitive conclusion in this matter.
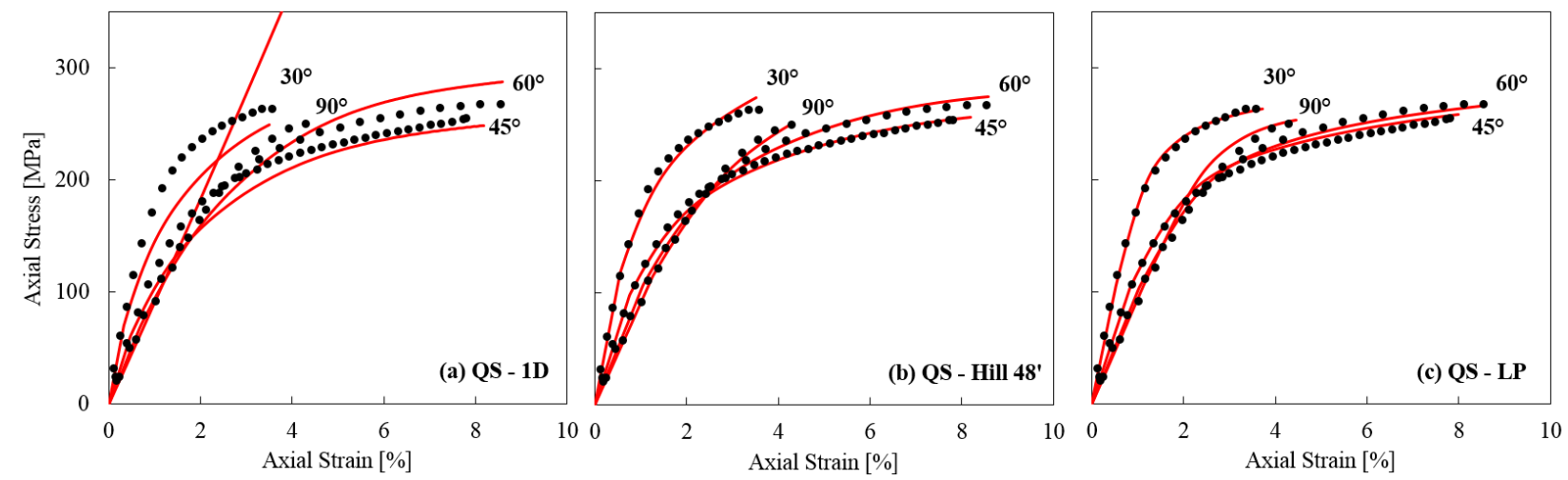

Fig.3. Quasi-static numerical simulations of off-axis compression tests compared to the experimental data reported in [13].
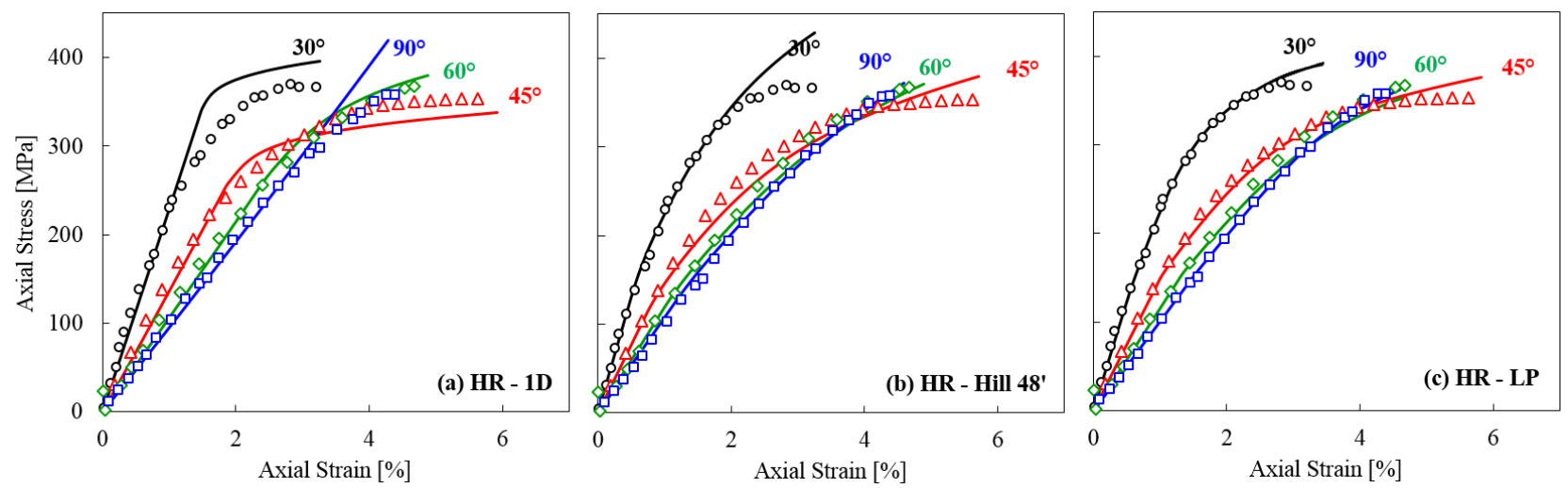

Fig.4. High-rate numerical simulations of off-axis compression tests compared to the experimental data reported in [13].
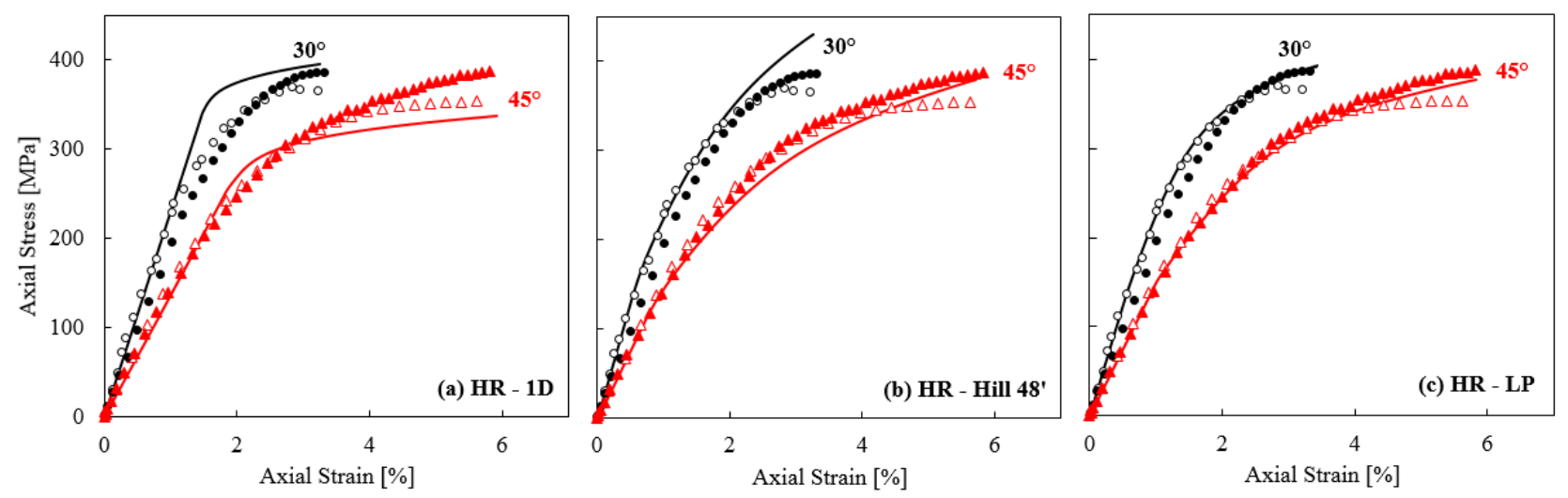

Fig.5. Off-axis compression tests reported in [13] depicted in hollow markers and in-house made SHPB tests on the same material plotted in solid markers compared to the numerical simulations.

\section{Concluding remarks}

Three different approaches to simulate the non-linear compressive behaviour of UD FRPs are presented. As a first assessment of such models numerical simulations of single elements were compared to off-axis quasi-static and high rate compressive stress-strain behaviour of UD laminates of IM7/8552 material reported in the literature. The 1D plasticity model proved to be able to reasonably predict the mechanical response of the material under high rates but further improvement would be needed in order to predict the quasi-static behaviour.
The other two approaches showed very good agreement between experimental and numerical behaviours, especially with the addition of new in-house high-rate tests at $30^{\circ}$ and $45^{\circ}$ orientations.

The authors would like to acknowledge the support of the Department of Engineering Science at the University of Oxford. As well as the valuable discussions with Dr. Jens Wiegand. 
Table 1. Elastic properties.

\begin{tabular}{|c|c|c|c|c|c|}
\hline$E_{1}[\mathrm{GPa}]$ & $E_{2}=E_{3}[\mathrm{GPa}]$ & $G_{12}=G_{13}[\mathrm{GPa}]$ & $G_{23}[\mathrm{GPa}]$ & $v_{12}=v_{13}$ & $v_{23}$ \\
\hline 154.486 & 9.003 & 5.068 & 2.150 & 0.32 & 0.30 \\
\hline
\end{tabular}

Table 2. Plasticity parameters for the $1 \mathrm{D}$ approach.

\begin{tabular}{|c|c|c|c|c|c|c|}
\hline \multicolumn{6}{|l|}{ Hardening constants and strain rate dependency } \\
\hline$\sigma_{0}[\mathrm{MPa}]$ & $Q_{1}[\mathrm{MPa}]$ & $C_{1}$ & $Q_{2}[\mathrm{GPa}]$ & $C_{2}$ & $k_{p}[\mathrm{~s}]$ & $r_{\mathrm{c}}[\mathrm{s}]$ \\
\hline 30 & 23.350 & 224.618 & 62.914 & 29.728 & 0.0325 & $10^{-5}$ \\
\hline
\end{tabular}

Table 3. Plasticity parameters for the Hill'48 approach.

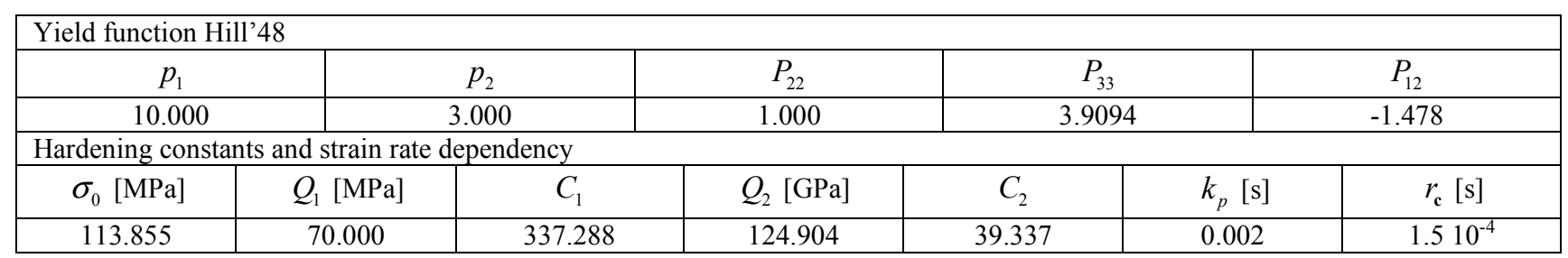

Table 4. Plasticity parameters for the LP approach.

\begin{tabular}{|c|c|c|c|c|c|c|}
\hline \multicolumn{7}{|c|}{ Yield function LP } \\
\hline \multicolumn{2}{|c|}{$\alpha_{f}$} & \multicolumn{2}{|l|}{$\beta$} & $\alpha_{g}$ & \multicolumn{2}{|c|}{$e$} \\
\hline \multicolumn{2}{|c|}{\begin{tabular}{l|l}
0.284 \\
\end{tabular}} & \multicolumn{2}{|l|}{0.985} & 0.228 & \multicolumn{2}{|c|}{0.480} \\
\hline \multicolumn{7}{|c|}{ Hardening constants and strain rate dependency } \\
\hline$\sigma_{0}[\mathrm{MPa}]$ & $Q_{1}[\mathrm{MPa}]$ & $C_{1}$ & $Q_{2}[\mathrm{GPa}]$ & $\mathrm{C}_{2}$ & $k_{p}[\mathrm{~s}]$ & $r_{\mathrm{c}}[\mathrm{s}]$ \\
\hline 38.355 & 30.567 & 154.300 & 22.333 & 22.183 & 0.0086 & $1.510^{-4}$ \\
\hline
\end{tabular}

\section{References}

1. R.K. Goldberg, K.S. Carney, P. Du Bois, C. Hoffarth, J. Harrington, R. Subramaniam, G. Blakenhorn, Theoretical Development of an Orthotropic Elasto-Plastic Generalized Composite Material Model in: 13th International LSDYNA Users Conference

2. C. Hoffarth, J. Harrington, R.D. Subramaniam, R.K. Goldberg, K.S. Carney, P. Du Bois, G. Blakenhorn, Verification and Validation of a Three-Dimensional Generalized Composite Material Model in: 13th International LS-DYNA Users Conference

3. A. Puck, M. Mannigel, Physically based non-linear stressstrain relations for the inter-fibre fracture analysis of FRP laminates, Composites Science and Technology, 67, 19551964 (2007)

4. M. Xie, D.F. Adams, A plasticity model for unidirectional composite materials and its applications in modeling composites testing, Composites Science and Technology, 54, 11-21 (1995)

5. G.M. Vyas, S.T. Pinho, P. Robinson, Constitutive modelling of fibre-reinforced composites with unidirectional plies using a plasticity-based approach, Composites Science and Technology, 71 1068-1074 (2011)

6. D.M. Thomson, B. Erice, H. Cui, J. Hoffmann, J. Wiegand, N. Petrinic, A Puck-based localisation plane theory for rate- and pressure-dependent constitutive modelling of unidirectional fibre-reinforced polymers, Composite Structures, 184 299-305 (2018)

7. R. Gerlach, C.R. Siviour, N. Petrinic, J. Wiegand, Experimental characterisation and constitutive modelling of RTM-6 resin under impact loading, Polymer, 49, 27282737 (2008)
8. W.M. Wang, L.J. Sluys, R. de Borst, Viscoplasticity for instabilities due to strain softening and strain-rate softening, International Journal for Numerical Methods in Engineering, 40, 3839-3864 (1997)

9. S. Eskandari, F.M. Andrade Pires, P.P. Camanho, A.T. Marques, Intralaminar damage in polymer composites in the presence of finite fiber rotation: Part I - Constitutive model, Composite Structures, 151, 114-126 (2016)

10. T. Børvik, Hopperstad, O.S., Berstad, T., Langseth, M., A computational model of viscoplasticity and ductile damage for impact and penetration, European Journal of Mechanics - A/Solids, 20, 685-712 (2001)

11. B. Erice, C.C. Roth, D. Mohr, Stress-state and strain-rate dependent ductile fracture of dual and complex phase steel, Mechanics of Materials, 116, 11-32 (2018)

12. G.R. Johnson, W.H. Cook, A Constitutive Model and Data for Metals Subjected to Large Strains, High Strain Rates and High Temperatures, in: 7th International Symposium on Ballistics, The Hague, pp. 541-547 1983

13. H. Koerber, J. Xavier, P.P. Camanho, High strain rate characterisation of unidirectional carbon-epoxy IM7-8552 in transverse compression and in-plane shear using digital image correlation, Mechanics of Materials, 42, 1004-1019 (2010)

14. H. Korber, Mechanical Response of Advanced Composites under High Strain Rates (Departamento de Engenharia Mecanica, Universidade do Porto, 2010)

15. D.M. Thomson, H. Cui, B. Erice, J. Hoffmann, J. Wiegand, N. Petrinic, Experimental and numerical study of strain-rate effects on the IFF fracture angle using a new efficient implementation of Puck's criterion, Composite Structures, 181, 25-335 (2017) 